Fixed Point Theory, 21(2020), No. 1, 211-220

DOI: $10.24193 /$ fpt-ro.2020.1.15

http://www.math.ubbcluj.ro/ nodeacj/sfptcj.html

\title{
A NOTE ON A RATIONAL FORM CONTRACTIONS WITH DISCONTINUITIES AT FIXED POINTS
}

\author{
E. KARAPINAR \\ Atilim University, Department of Mathematics,06836, Incek, Ankara, Turkey \\ and \\ Department of Medical Research, China Medical University, 40402, Taichung, Taiwan \\ E-mail: erdalkarapinar@yahoo.com
}

\begin{abstract}
In this paper, we investigate one of the classical problems of the metric fixed point theory: Whether there is a contraction condition which does not force the mapping to be continuous at the fixed point. We propose a contraction conditions in rational form that has a unique fixed
\end{abstract} point but not necessarily continuous at the given fixed point.

Key Words and Phrases: Discontinuity, fixed point theorems, metric space.

2010 Mathematics Subject Classification: 47H10, 54H10, 54H25.

\section{REFERENCES}

[1] M. Arshad, E. Karapinar, J. Ahmad, Some unique fixed point theorems for rational contractions in partially ordered metric spaces, J. Inequal. Appl., 2013, 2013:248.

[2] R.K. Bisht, R.P. Pant, A remark on discontinuity at fixed point, J. Math. Anal. Appl., 445(2017), 1239-1242.

[3] B.K. Dass, S. Gupta, An extension of Banach contraction principle through rational expressions, Indian J. Pure Appl. Math., 6(1975), 1455-1458.

[4] S. Chandok, E. Karapinar, Common fixed point of generalized rational type contraction mappings in partially ordered metric spaces, Thai J. Math., 11(2013), no. 2. 251-260.

[5] J. Jachymski, Common fixed point theorems for some families of maps, Indian J. Pure Appl. Math., 25(1994), 925-937.

[6] J. Jachymski, Equivalent conditions and Meir-Keeler type theorems, J. Math. Anal. Appl., 194(1995), 293-303.

[7] D.S. Jaggi, Some unique fixed point theorems, Indian J. Pure Appl. Math., 8(1977), 223-230.

[8] R. Kannan, Some results on fixed points II, Amer. Math. Monthly, 76(1969), 405-408.

[9] E. Karapinar, A. Dehici, N. Redjel, On some fixed points of $(\alpha-\psi)$-contractive mappings with rational expressions, J. Nonlinear Sci. Appl., 10(2017), 1569-1581.

[10] J. Matkowski, Integrable solutions of functional equations, Dissertationes Math., 127(1975), $1-68$.

[11] A. Meir, E. Keeler, A theorem on contraction mappings, J. Math. Anal. Appl., 28(1969), 326329 .

[12] Z. Mustafa, E. Karapinar, H. Aydi, A discussion on generalized almost contractions via rational expressions in partially ordered metric spaces, J. Inequal. Appl., 2014, 2014:219.

[13] R.P. Pant, Discontinuity and fixed points, J. Math. Anal. Appl., 240(1999), 284-289. 
[14] B.E. Rhoades, A comparison of various definitions of contractive mappings, Trans. Amer. Math. Soc., 226(1977), 257-290.

[15] B.E. Rhoades, Contractive definitions and continuity, Contemp. Math., 72(1988), 233-245.

Received: June 7, 2018; Accepted: October 31, 2018. 